Supporting Information for

\title{
Solvent and Substituent Effects on Aggregation Behavior of Surface Active Ionic Liquids with Aromatic Counterions and Dispersion of Carbon Nanotubes in their Hexagonal Liquid-Crystalline Phase
}

\author{
Wenwen $\mathrm{Xu},{ }^{a, b}$ Qiwen Yin, ${ }^{c}$ Yan'an Gao, ${ }^{d}$ and Li Yu ${ }^{a, b^{*}}$ \\ ${ }^{a}$ Key Laboratory of Colloid and Interface Chemistry, Shandong University, Ministry of Education, Jinan 250100, \\ PR China. \\ ${ }^{b}$ School of Chemistry and Chemical Engineering, Qufu Normal University, Qufu, 273165,PR China. \\ ${ }^{c}$ China Research Institute of Daily Chemical Industry, Taiyuan 030001, PR China. \\ ${ }^{d}$ Dalian Institute of Chemical Physics, Chinese Academy of Sciences, Dalian 116023, PR China.
}

Corresponding author:

Prof. Dr. Li Yu

Phone number: $+86-531-88364807$

Fax number: $+86-531-88564750$

E-mail address: ylmlt@sdu.edu.cn 


\section{${ }^{1} \mathrm{H}$ NMR spectroscopy for $m-\mathrm{C}_{12} \mathrm{mimHB}$ and $p-\mathrm{C}_{12} \mathrm{mimHB}$}

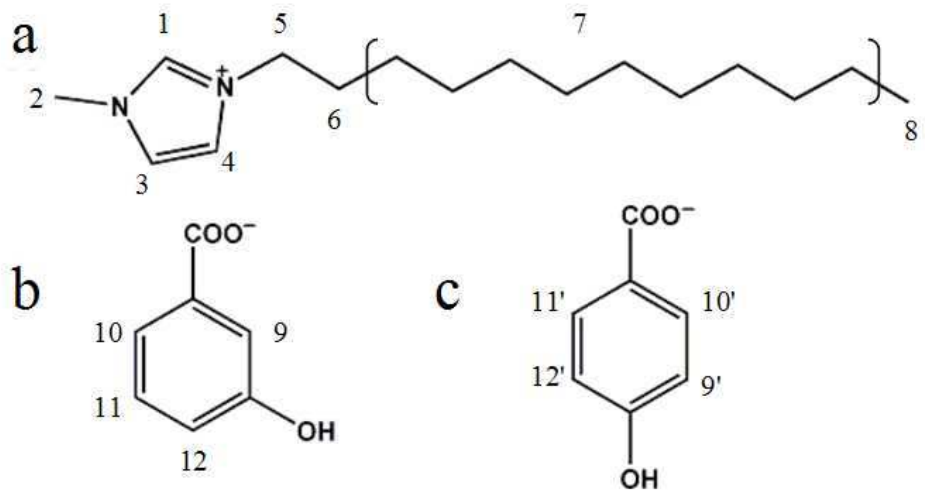

Scheme 1 Chemical structures of (a) $\mathrm{C}_{12} \mathrm{mim}^{+}$, (b) $m-\mathrm{HB}^{-}$and (c) $p$ - $\mathrm{HB}^{-}$.

For $m-\mathrm{C}_{12} \mathrm{mimHB},{ }^{1} \mathrm{H} \mathrm{NMR}\left(\mathrm{CDCl}_{3}, \delta / \mathrm{ppm}\right): 0.87\left(\mathrm{t}, 3 \mathrm{H}, \mathrm{H}_{8}\right), 1.04-1.32\left(\mathrm{~d}, 18 \mathrm{H}, \mathrm{H}_{7}\right)$, $1.54\left(\mathrm{t}, 2 \mathrm{H}, \mathrm{H}_{6}\right), 3.82\left(\mathrm{~s}, 3 \mathrm{H}, \mathrm{H}_{2}\right), 3.88\left(\mathrm{t}, 2 \mathrm{H}, \mathrm{H}_{5}\right), 6.76\left(\mathrm{~d}, 1 \mathrm{H}\right.$, arom. $\left.\mathrm{H}_{12}\right), 7.01(\mathrm{t}, 1 \mathrm{H}$, arom. $\left.\mathrm{H}_{11}\right), 7.14\left(\mathrm{~m}, 2 \mathrm{H}, \mathrm{H}_{3}\right.$ and $\left.\mathrm{H}_{4}\right), 7.26\left(\mathrm{~s}, 1 \mathrm{H}\right.$, arom. $\left.\mathrm{H}_{9}\right), 7.37\left(\mathrm{~d}, 1 \mathrm{H}\right.$, arom. $\left.\mathrm{H}_{10}\right)$, $9.43\left(\mathrm{~s}, 1 \mathrm{H}, \mathrm{H}_{1}\right)$. For $p-\mathrm{C}_{12} \operatorname{mimHB},{ }^{1} \mathrm{H}$ NMR $\left(\mathrm{CDCl}_{3}, \delta / \mathrm{ppm}\right): 0.86\left(\mathrm{t}, 3 \mathrm{H}, \mathrm{H}_{8}\right)$, 1.20-1.24 (d, 18H, $\left.\mathrm{H}_{7}\right), 1.68\left(\mathrm{t}, 2 \mathrm{H}, \mathrm{H}_{6}\right), 3.72\left(\mathrm{~s}, 3 \mathrm{H}, \mathrm{H}_{2}\right), 4.00\left(\mathrm{t}, 2 \mathrm{H}, \mathrm{H}_{5}\right), 6.73(\mathrm{~d}, 2 \mathrm{H}$, arom. $\mathrm{H}_{9^{\prime}}$ and arom. $\left.\mathrm{H}_{12^{\prime}}\right), 7.19\left(\mathrm{~m}, 2 \mathrm{H}, \mathrm{H}_{3}\right.$ and $\left.\mathrm{H}_{4}\right), 7.75\left(\mathrm{~m}, 2 \mathrm{H}\right.$, arom. $\mathrm{H}_{10^{\prime}}$ and arom. $\left.\mathrm{H}_{11^{\prime}}\right), 9.42\left(\mathrm{~s}, 1 \mathrm{H}, \mathrm{H}_{1}\right)$. 

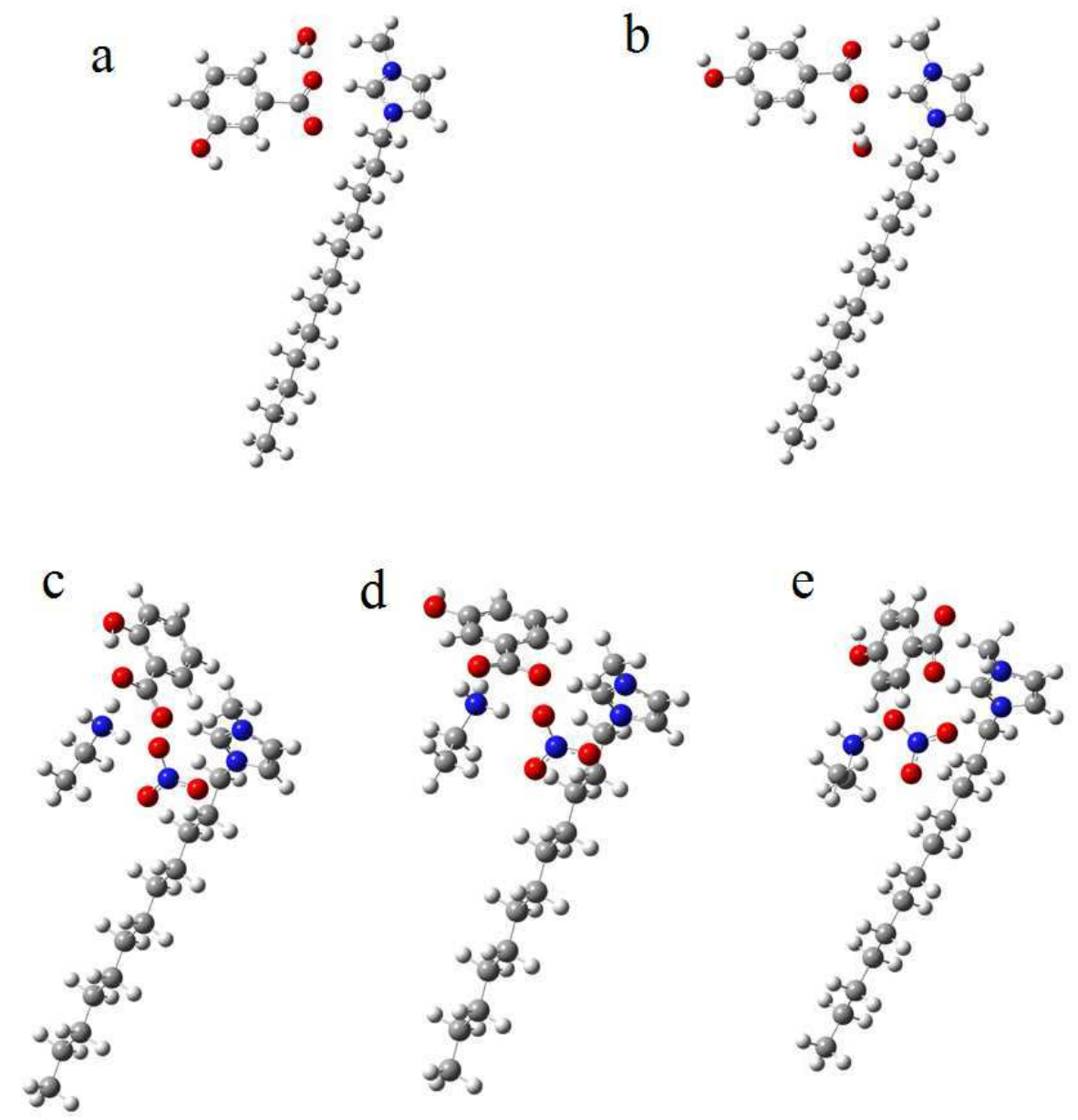

Figure S1 Geometries optimized at the B3LYP/6-31G(d,p) level: (a) $m-\mathrm{C}_{12} \mathrm{mimHB} / \mathrm{H}_{2} \mathrm{O}$, (b) $p$ - $\mathrm{C}_{12} \mathrm{mimHB} / \mathrm{H}_{2} \mathrm{O}$, (c) $\mathrm{C}_{12} \mathrm{mimSal} / \mathrm{EAN}$, (d) $m-\mathrm{C}_{12} \operatorname{mimHB} / \mathrm{EAN}$, (e) $p-\mathrm{C}_{12} \mathrm{mimHB} / \mathrm{EAN}$.

Color code for atoms: blue, nitrogen; red, oxygen; dark gray, carbon; light gray, hydrogen.

Table S1 Interaction energy ( $\left.-E_{\text {int }}\right)$ of SAILs with $\mathrm{H}_{2} \mathrm{O}$ and EAN.

\begin{tabular}{ccc}
\hline SAILs & $-E_{\text {int }}\left(\mathrm{kJ} \cdot \mathrm{mol}^{-1}\right)$ \\
\hline $\mathrm{C}_{12}$ mimSal & $110.47^{a, c}$ & $737.98^{b}$ \\
$m-\mathrm{C}_{12} \operatorname{mimHB}$ & $71.60^{a}$ & $610.56^{b}$ \\
$p-\mathrm{C}_{12} \operatorname{mimHB}$ & $70.49^{a}$ & $607.53^{b}$ \\
\hline${ }^{a}$ with $\mathrm{H}_{2} \mathrm{O}$. & \\
${ }^{b}$ with EAN. & \\
${ }^{c}$ reported in ref. 36.
\end{tabular}



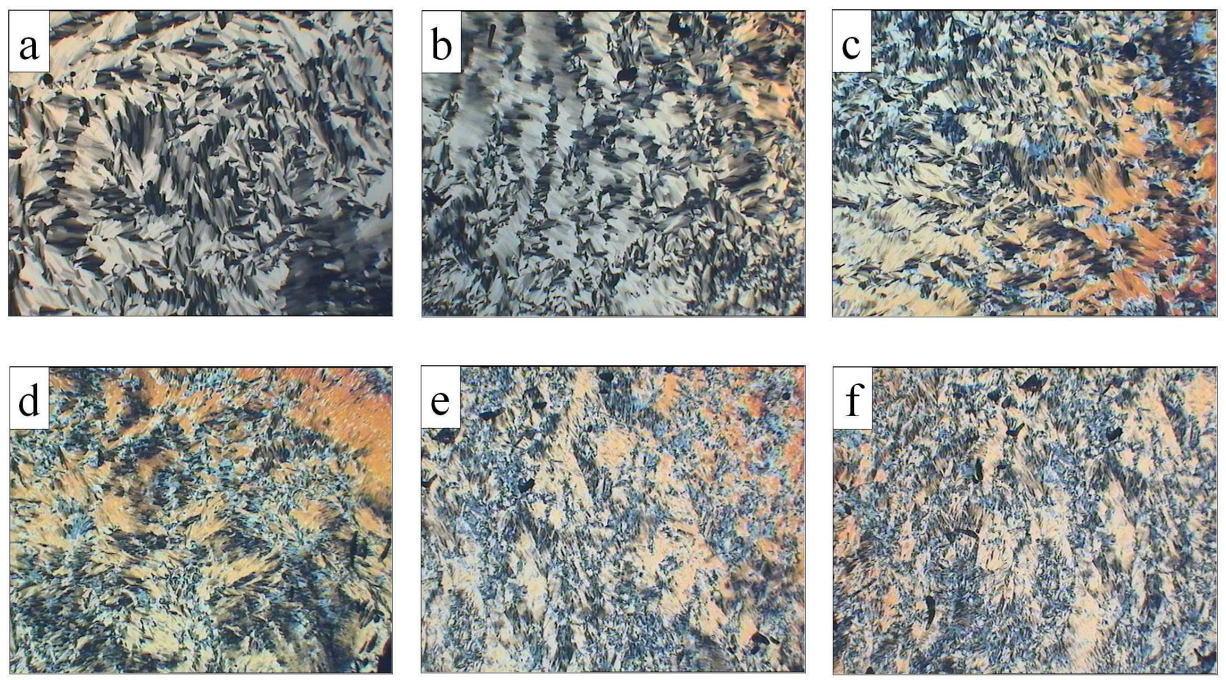

Figure S2 POM images of the $\mathrm{H}_{1}$ phase formed by the $m-\mathrm{C}_{12}$ mimHB/ $\mathrm{H}_{2} \mathrm{O}$ system with different concentration at $25^{\circ} \mathrm{C}$ : (a) $55 \mathrm{wt} \%$, (b) $60 \mathrm{wt} \%$, (c) $65 \mathrm{wt} \%$, (d) $70 \mathrm{wt} \%$, (e) $80 \mathrm{wt} \%$ and (f) $90 \mathrm{wt} \%$.
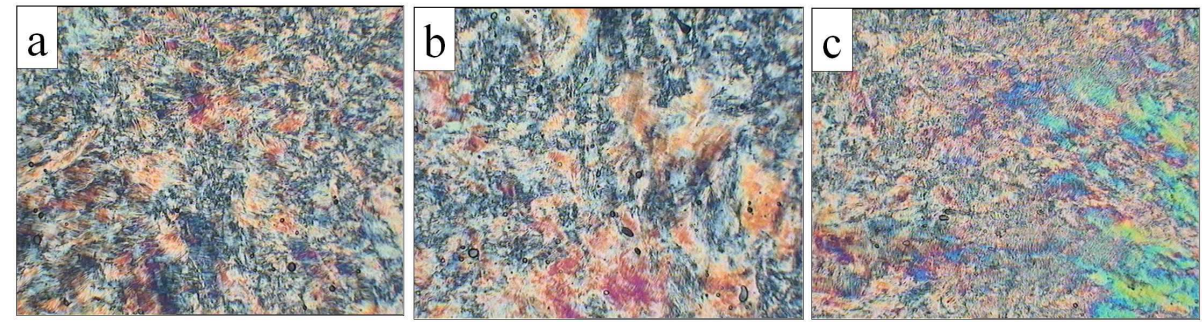

Figure S3 POM images for the $p-\mathrm{C}_{12} \mathrm{mimHB} / \mathrm{H}_{2} \mathrm{O}$ system with different concentration of $p-\mathrm{C}_{12} \operatorname{mimHB}$ at $25^{\circ} \mathrm{C}$ : (a) $55 \mathrm{wt} \%$, (b) $65 \mathrm{wt} \%$, (c) $75 \mathrm{wt} \%$.
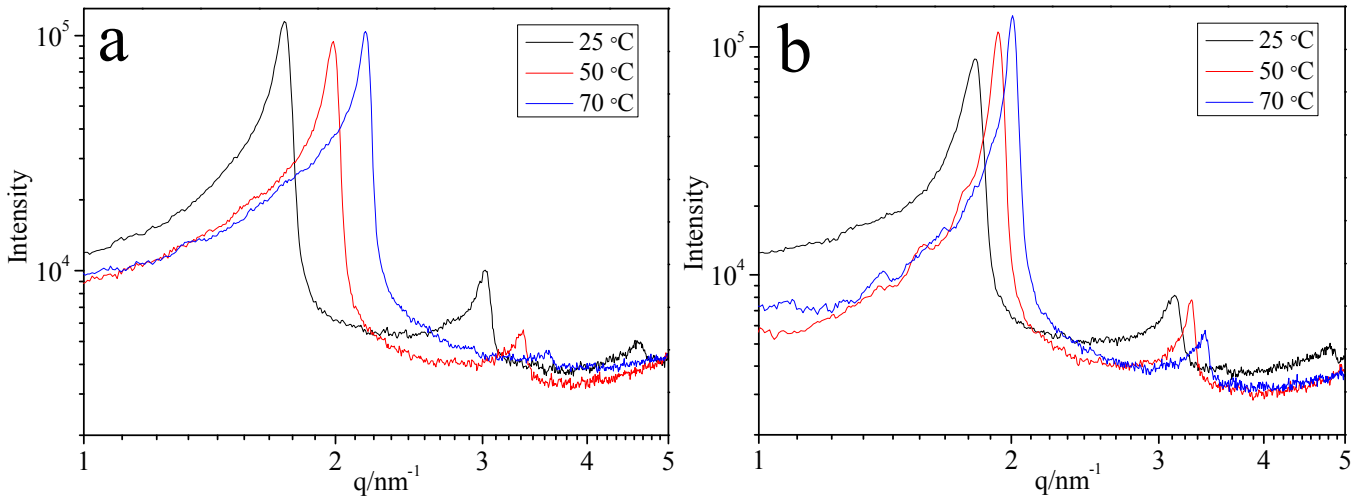

Figure S4 SAXS curves of the $\mathrm{H}_{1}$ phase formed by $60 \mathrm{wt} \% m-\mathrm{C}_{12} \operatorname{mimHB}$ (a) and $p-\mathrm{C}_{12} \mathrm{mimHB}$ (b) in aqueous solutions at different temperature. 

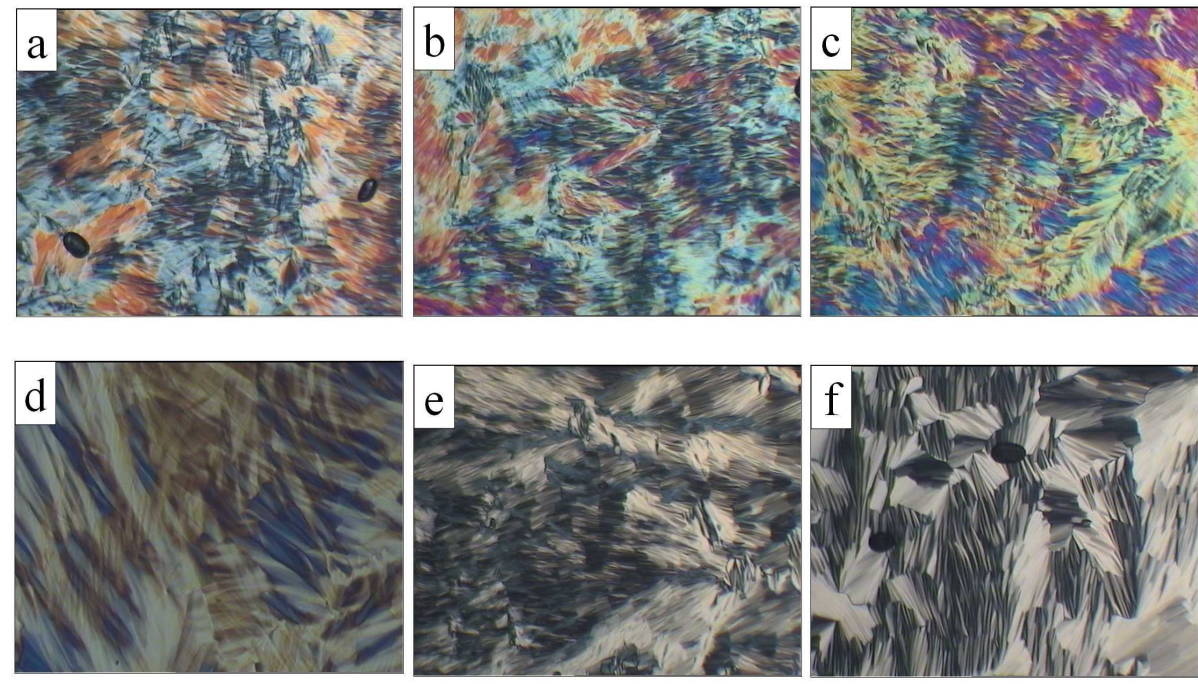

Figure S5 POM images of the $\mathrm{H}_{1}$ phase formed by the $60 \mathrm{wt} \% m-\mathrm{C}_{12} \mathrm{mimHB} / \mathrm{H}_{2} \mathrm{O}$ system at different temperature: (a) $25^{\circ} \mathrm{C}$, (b) $50^{\circ} \mathrm{C}$, (c) $70{ }^{\circ} \mathrm{C}$, and the $60 \mathrm{wt} \% p-\mathrm{C}_{12} \mathrm{mimHB} / \mathrm{H}_{2} \mathrm{O}$ system at different temperature: (d) $25^{\circ} \mathrm{C}$, (e) $50^{\circ} \mathrm{C}$, (f) $70^{\circ} \mathrm{C}$.

Table S2 Structural parameters for the $\mathrm{H}_{1}$ phase formed by SAILs in EAN at $25^{\circ} \mathrm{C}$.

\begin{tabular}{ccccc}
\hline & $a_{0}(\AA)$ & $d_{\mathrm{H}}(\AA)$ & $d_{\mathrm{W}}(\AA)$ & $a_{\mathrm{s}}\left(\AA^{2}\right)$ \\
\hline $\begin{array}{c}m-\mathrm{C}_{12} \mathrm{mimHB} \\
(80 \mathrm{wt} \%)\end{array}$ & 32.39 & 13.21 & 1.96 & 46.05 \\
$p-\mathrm{C}_{12} \mathrm{mimHB}$ & 30.06 & 14.97 & 0.12 & 46.78 \\
$(90 \mathrm{wt} \%)$ & & & & \\
\hline
\end{tabular}




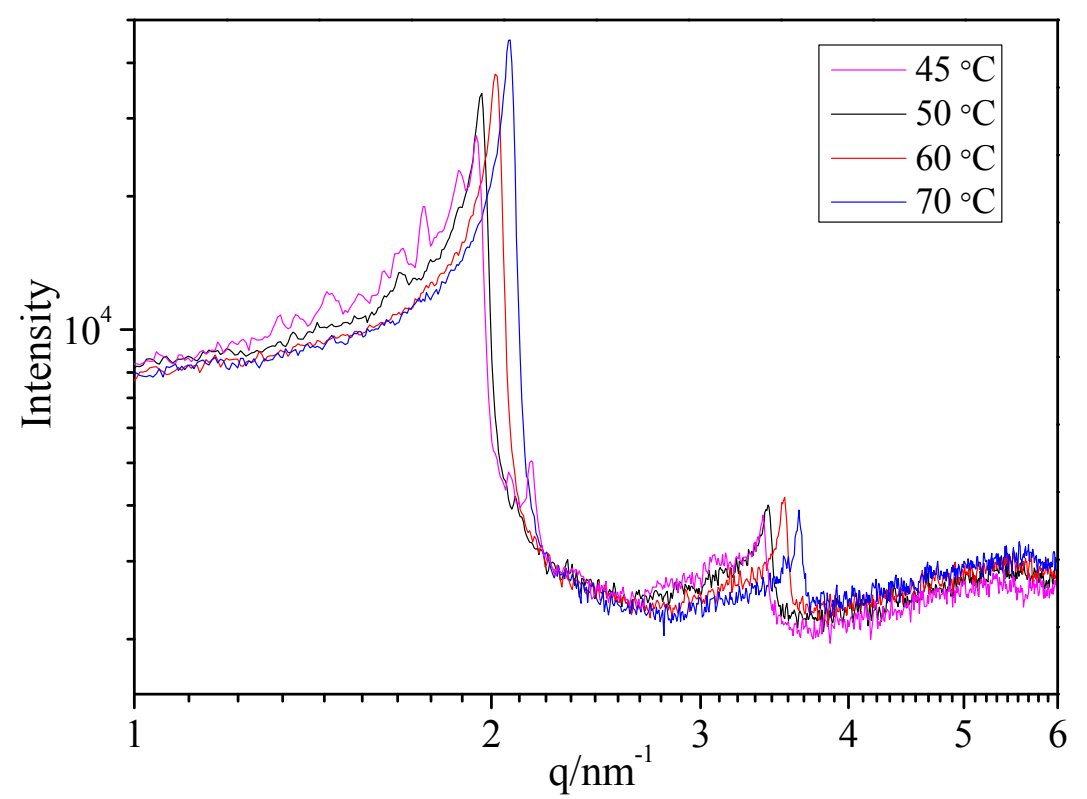

Figure S6 SAXS curves of the $\mathrm{H}_{1}$ phase formed by $80 \mathrm{wt} \% m-\mathrm{C}_{12} \operatorname{mimHB}$ in EAN at different temperature.
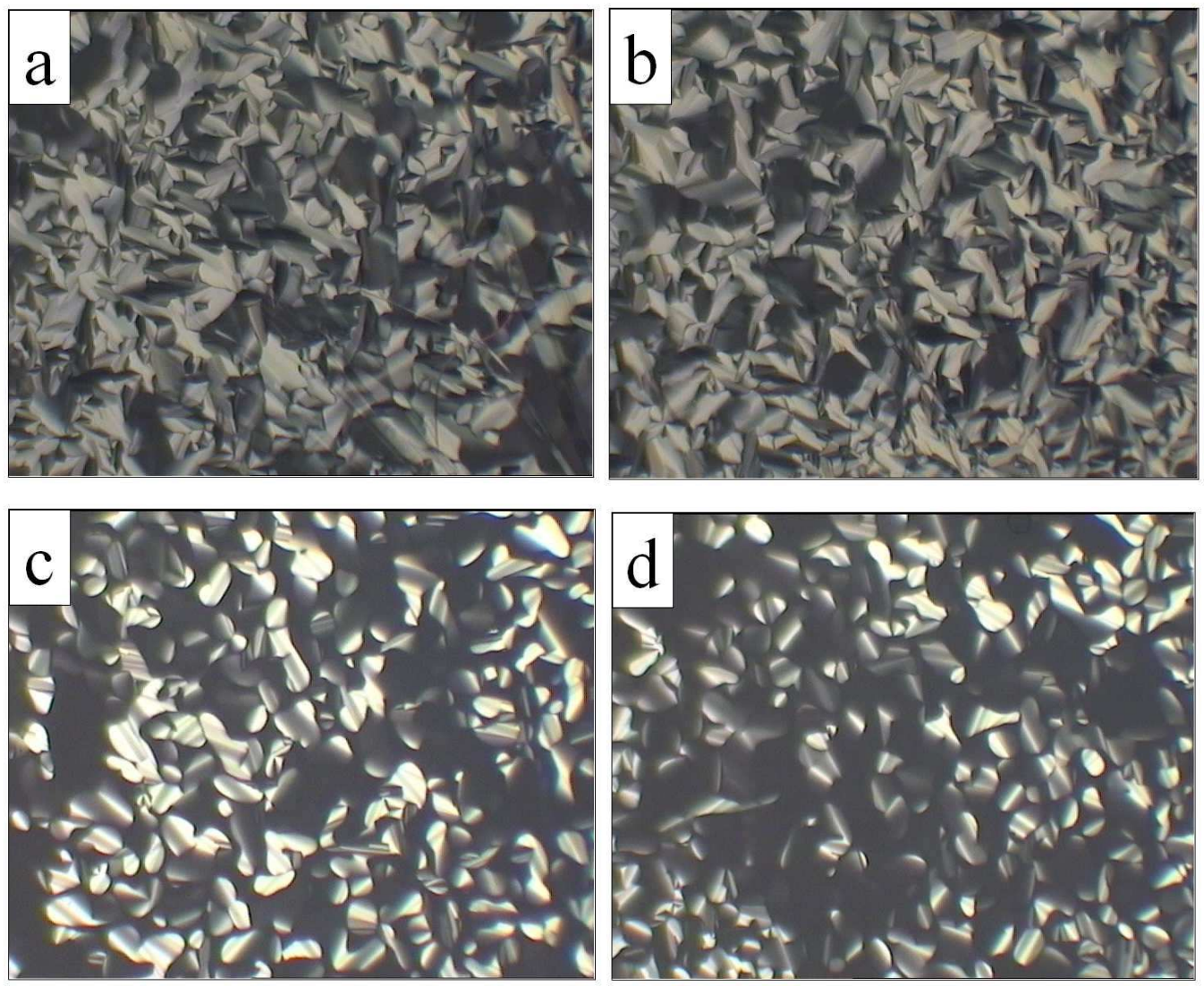

Figure S7 POM images of the $\mathrm{H}_{1}$ phase formed by $80 \mathrm{wt} \% \mathrm{~m}$ - $\mathrm{C}_{12}$ mimHB in EAN at different temperature: (a) $45^{\circ} \mathrm{C}$, (b) $50^{\circ} \mathrm{C}$, (c) $60{ }^{\circ} \mathrm{C}$, (d) $70{ }^{\circ} \mathrm{C}$. 

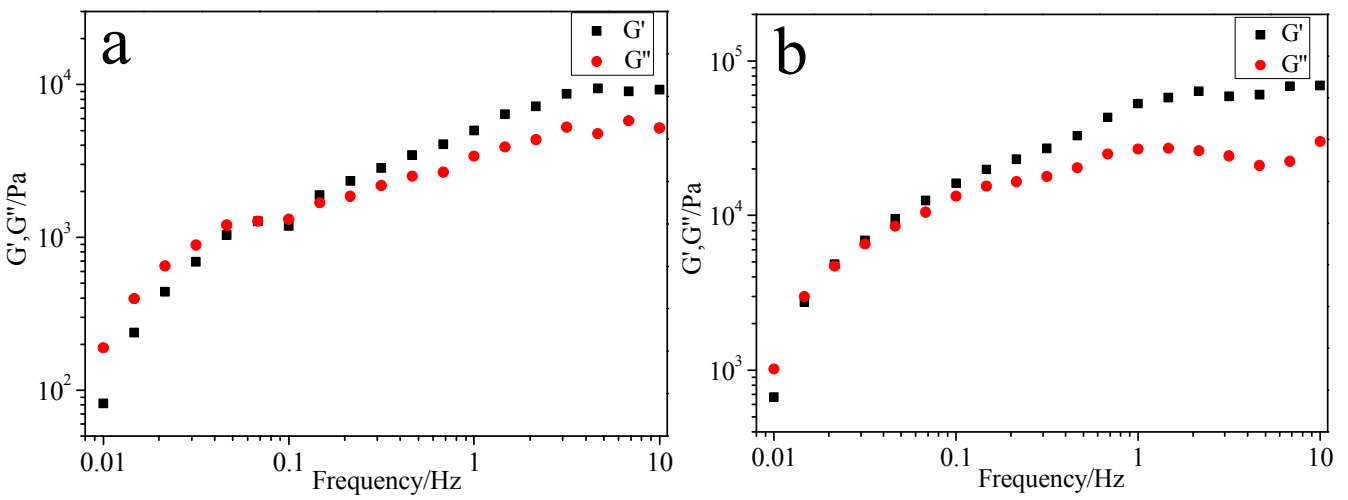

Figure S8 Frequency sweep curves for the $\mathrm{H}_{1}$ phases formed by $80 \mathrm{wt} \% \mathrm{~m}-\mathrm{C}_{12} \operatorname{mimHB}$ (a) and $90 \mathrm{wt} \% p-\mathrm{C}_{12} \operatorname{mimHB}(\mathrm{b})$ in EAN.
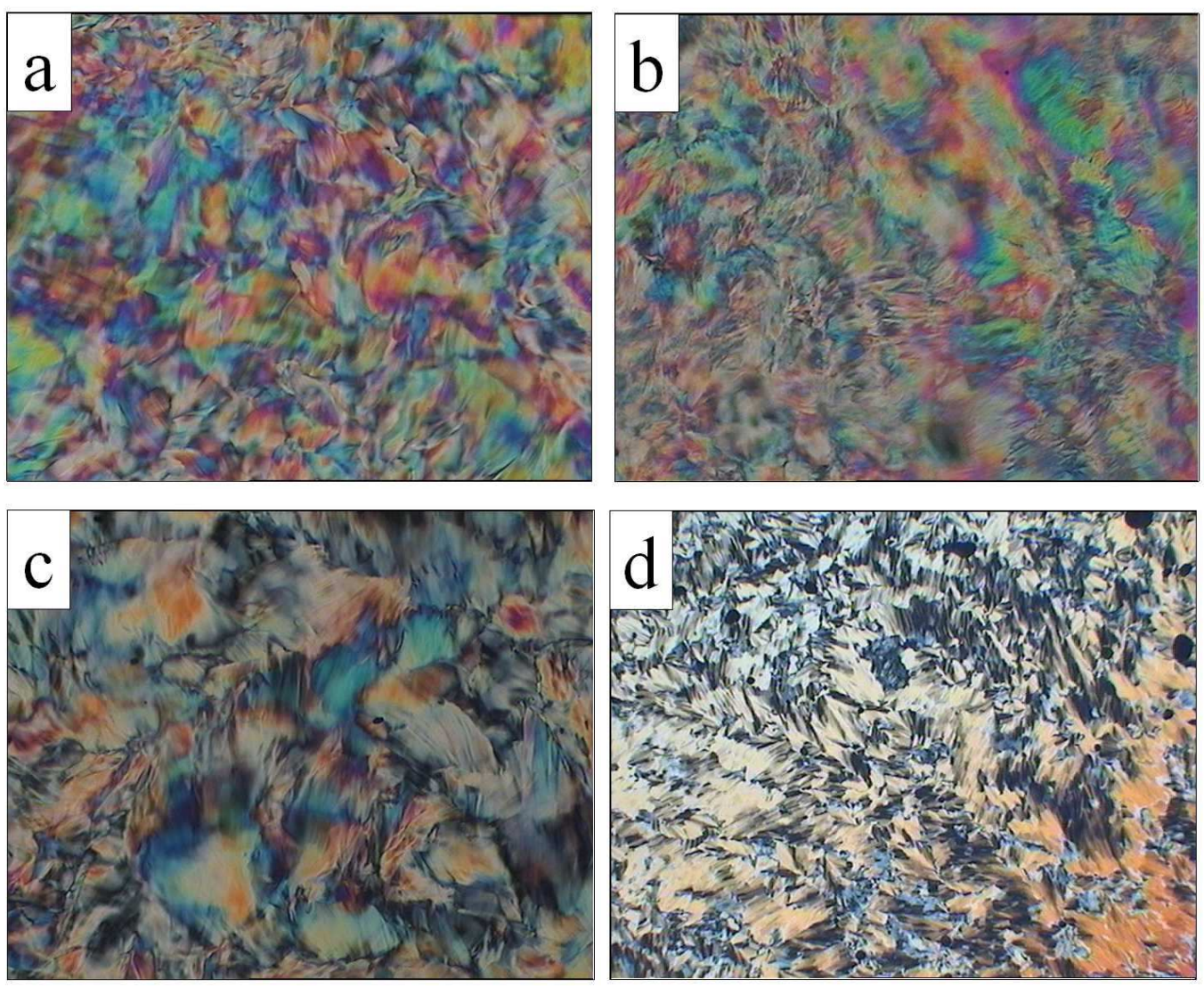

Figure S9 POM images of the $\mathrm{H}_{1}$ phase formed by $60 \mathrm{wt} \% m-\mathrm{C}_{12} \mathrm{mimHB}$ in aqueous solution with different concentration of MWCNTs at $25{ }^{\circ} \mathrm{C}$ : (a) $0.01 \mathrm{wt} \%$, (b) $0.05 \mathrm{wt} \%$, (c) $0.10 \mathrm{wt} \%$, (d) $0.20 \mathrm{wt} \%$. 
Table S3 Structural parameters of $\mathrm{H}_{1}$ phase formed by $60 \mathrm{wt} \% \mathrm{C}_{12}$ mimHB at $25^{\circ} \mathrm{C}$ in the presence of different concentrations of MWCNTs.

\begin{tabular}{cccccc}
\hline & MWCNTs $(\mathrm{wt} \%)$ & $a_{0}(\AA)$ & $d_{\mathrm{H}}(\AA)$ & $d_{\mathrm{W}}(\AA)$ & $a_{\mathrm{s}}\left(\AA^{2}\right)$ \\
\hline$m-\mathrm{C}_{12}$ mimHB & 0.00 & 35.32 & 15.37 & 4.58 & 45.57 \\
& 0.01 & 35.90 & 15.62 & 4.66 & 44.84 \\
& 0.05 & 36.32 & 15.80 & 4.72 & 44.33 \\
& 0.10 & 36.53 & 15.89 & 4.75 & 44.08 \\
0.20 & 37.85 & 16.47 & 4.91 & 42.53 \\
& 0.00 & 35.32 & 15.37 & 4.58 & 45.57 \\
& 0.01 & 36.74 & 15.99 & 4.76 & 43.80 \\
& 0.05 & 37.40 & 16.27 & 4.86 & 43.05 \\
& 0.10 & 39.27 & 17.09 & 5.09 & 40.98 \\
& 0.20 & 40.54 & 17.63 & 5.28 & 39.73 \\
\hline
\end{tabular}
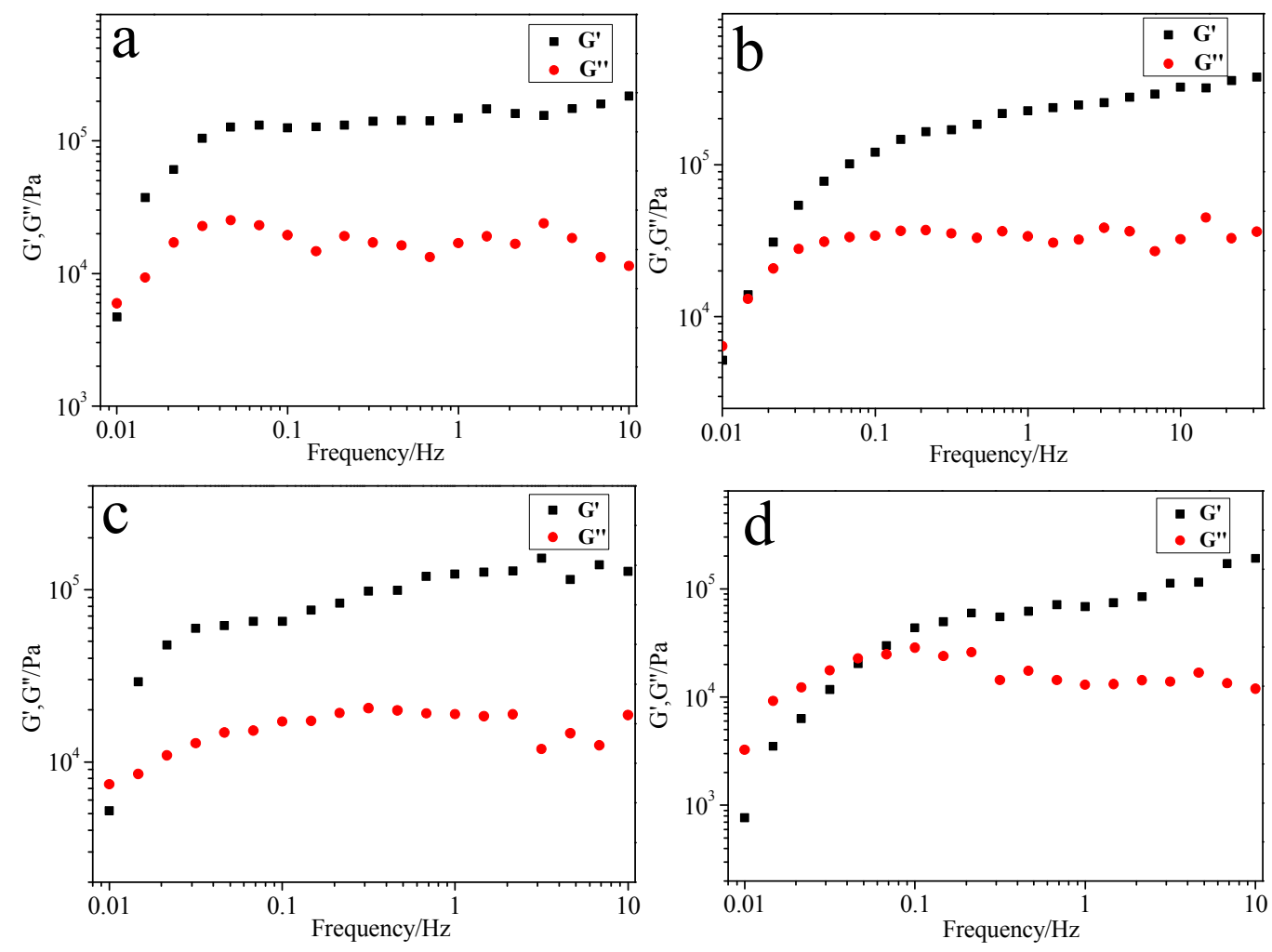

Figure S10 Frequency sweep curves for MWCNTs $/ m-\mathrm{C}_{12}$ mimHB LLC composites with different amount of MWCNTs: (a) $0.01 \mathrm{wt} \%$, (b) $0.05 \mathrm{wt} \%$, (c) $0.10 \mathrm{wt} \%$, (d) $0.20 \mathrm{wt} \%$. 

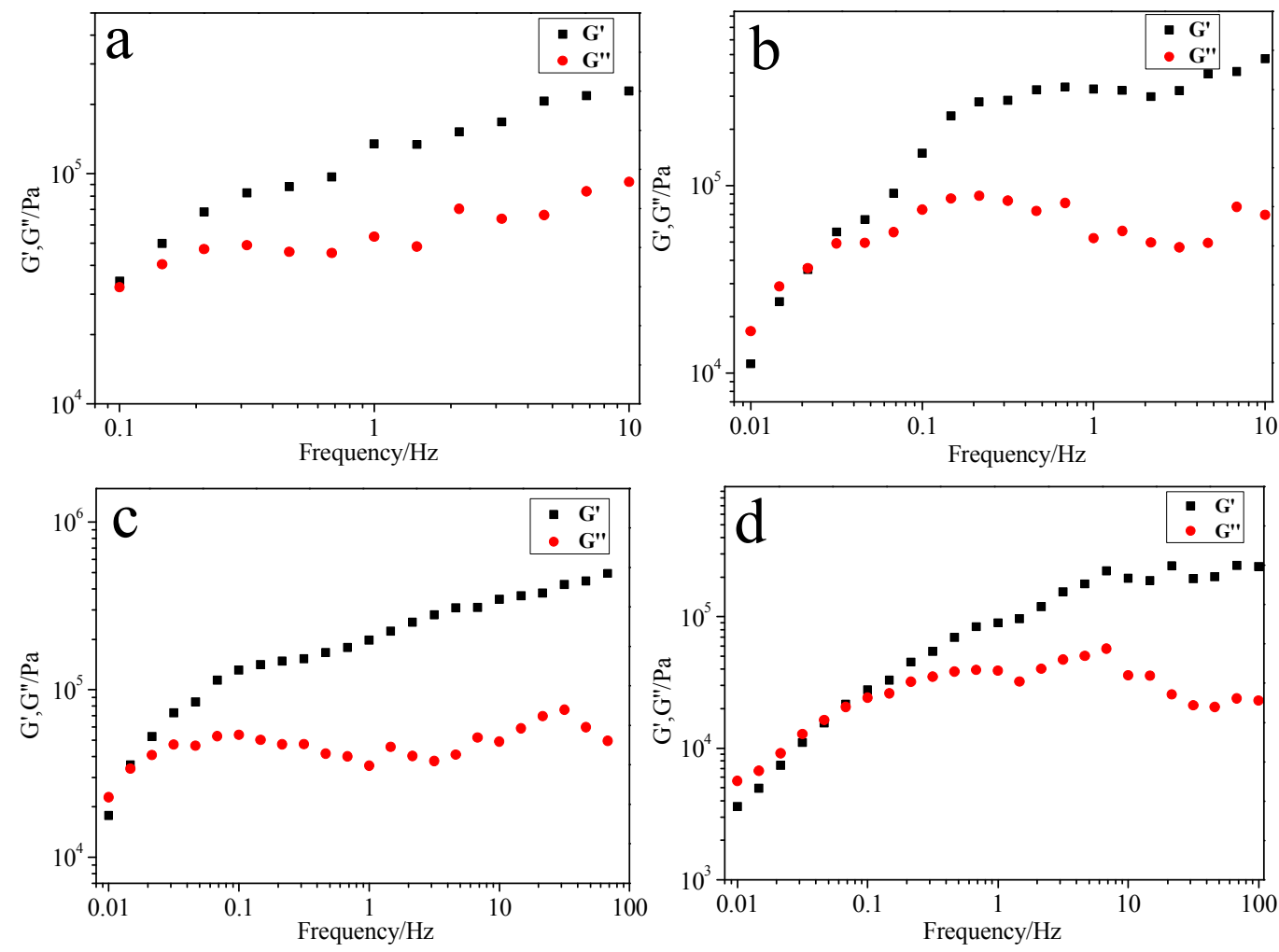

Figure S11 Frequency sweep lines for the $\mathrm{H}_{1}$ phase formed by $60 \mathrm{wt} \% m-\mathrm{C}_{12}$ mimHB in aqueous solution with different concentration of MWCNTs: (a) $0.01 \mathrm{wt} \%$, (b) $0.05 \mathrm{wt} \%$, (c) $0.10 \mathrm{wt} \%$,

(d) $0.20 \mathrm{wt} \%$.
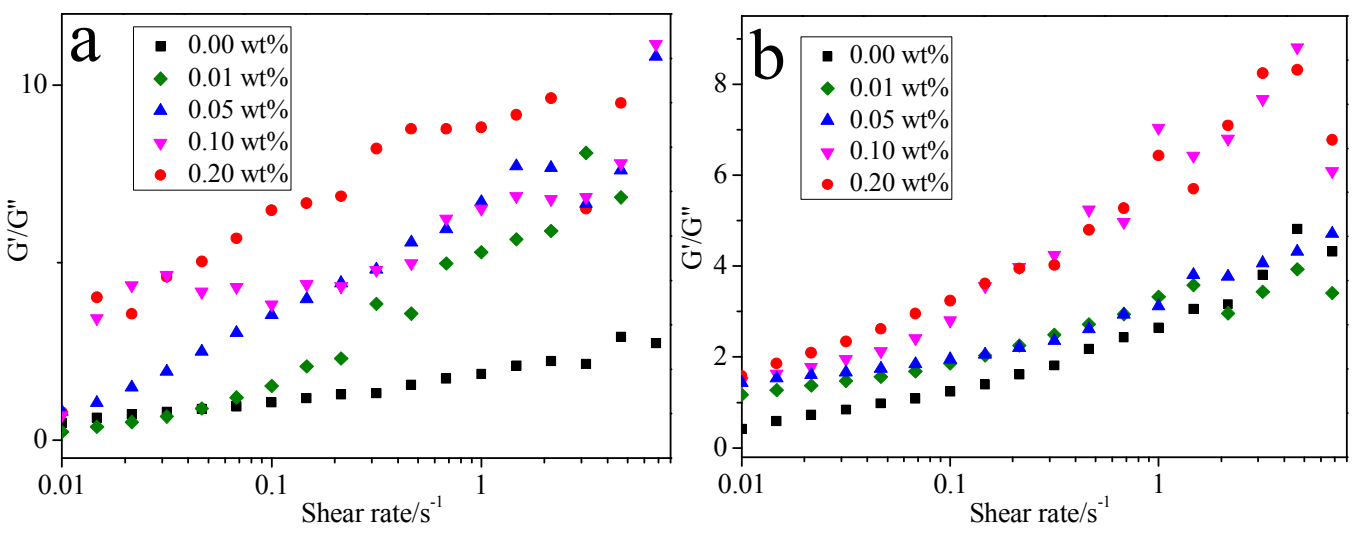

Figure $\mathrm{S} 12 G^{\prime} / G^{\prime \prime}$ values for the $\mathrm{H}_{1}$ phase formed by $60 \mathrm{wt} \%$ SAILs in aqueous solution with different concentration of MWCNTs: (a) $m-\mathrm{C}_{12} \operatorname{mimHB}$ and (b) $p-\mathrm{C}_{12}$ mimHB. 